\title{
Modeling the Black Hole Excision Problem
}

\author{
B. Szilágyi ${ }^{1,2}, \mathrm{H}-\mathrm{O}$. Kreiss ${ }^{2,3}$, and J. Winicour ${ }^{1,2}$ \\ ${ }^{1}$ Department of Physics and Astronomy, University of Pittsburgh, Pittsburgh, Pennsylvania 15260 \\ ${ }^{2}$ Albert Einstein Institute, Max Planck Gesellschaft, Am Mühlenberg 1, D-14476 Golm, Germany \\ ${ }^{3}$ NADA, Royal Institute of Technology, 10044 Stockholm, Sweden
}

(Dated: Dec 21, 2004)

\begin{abstract}
We analyze the excision strategy for simulating black holes. The problem is modeled by the propagation of quasi-linear waves in a 1-dimensional spatial region with timelike outer boundary, spacelike inner boundary and a horizon in between. Proofs of well-posed evolution and boundary algorithms for a second differential order treatment of the system are given for the separate pieces underlying the finite difference problem. These are implemented in a numerical code which accurately and stably simulates the quasi-linear excision problem. Excitation of long wavelength exponential modes, which are latent in the problem, are suppressed using conservation laws for the discretized system. The techniques are designed to apply directly to recent codes for the Einstein equations based upon the harmonic formulation.
\end{abstract}

PACS numbers: $\quad$ 04.25.Dm, 97.60.Lf, 04.20.Ex, 04.30.Db, 95.30.Sf

\section{INTRODUCTION}

Two computational difficulties in simulating the emission of gravitational waves from black hole interactions are the existence of a singularities inside the black holes and a consistent treatment of the outer boundary so that the waveform of the gravitational radiation can be accurately extracted. Fortunately, the observable waves emanate only from the region outside the black holes and can be simulated, without loss of physical content, in a domain from which the singularities have been excised by introducing inner boundaries inside the black holes. However, the treatment of this domain by Cauchy evolution has had limited success. We present here a quasi-linear model excision problem whose mathematical analysis is simple enough to reveal some underlying computational pitfalls and the means to avoid them. In particular, we show that a successful global treatment of this model excision problem can be obtained by (i) blending together two evolution algorithms, one of which is well-posed near the outer boundary and the other well-posed near the inner boundary, where a superluminal evolution is employed, and (ii) incorporating discrete conservation laws which control spurious exponential growth. The results are aimed at numerical formulations of the Einstein equation as second order quasi-linear wave equations, such as in recent harmonic approaches [1, 2, 3]. Items (i) and (ii) above were also found necessary, although in quite different form, in a model excision problem based upon a first order symmetric hyperbolic formulation [4], which suggests that they may be essential ingredients of any successful treatment.

The problem can be illustrated by considering the propagation of scalar waves on the fixed background geometry of a spherically symmetric Schwarzschild black hole. A choice of coordinates commonly adopted in numerical work for excising the singular region are ingoing Eddington-Finkelstein coordinates $x^{\alpha}=(t, r, \theta, \phi)$, in which the Schwarzschild spacetime metric $g_{\alpha \beta}$ is given by

$$
\begin{aligned}
g_{\alpha \beta} d x^{\alpha} d x^{\beta}= & -\left(1-\frac{2 M}{r}\right) d t^{2}+\frac{4 M}{r} d t d r+\left(1+\frac{2 M}{r}\right) d r^{2} \\
& +r^{2}\left(d \theta^{2}+\sin ^{2} \theta d \phi^{2}\right) .
\end{aligned}
$$

Here, the spacetime is foliated by the spacelike Cauchy hypersurfaces $t=$ const, which are used to carry out the numerical evolution. The radial coordinate extends from the location of the singularity at $r=0$ to the asymptotic region $r \rightarrow \infty$, to which the radiation propagates. The black hole is located at $r=2 M$. In the excision strategy, the simulation is carried out in the region $R_{1} \leq r \leq R_{2}$, where the inner boundary satisfies $0<R_{1}<2 M$ and the outer boundary (introduced for the purpose of a finite coordinate range) satisfies $R_{2}>>2 M$.

The covariant wave equation for a scalar field $u$ is

$$
\frac{1}{\sqrt{-g}} \partial_{\alpha}\left(\sqrt{-g} g^{\alpha \beta} \partial_{\beta} u\right)=0
$$

where $g=\operatorname{det}\left(g_{\alpha \beta}\right)$ and $g^{\alpha \beta}$ is the inverse metric. In the above coordinates, it takes the explicit form

$$
-\left(1+\frac{2 M}{r}\right) \partial_{t}^{2} u+\frac{4 M}{r} \partial_{t} \partial_{r} u+\left(1-\frac{2 M}{r}\right) \partial_{r}^{2} u+\frac{1}{r^{2}}\left(\partial_{\theta}^{2}+\frac{1}{\sin ^{2} \theta} \partial_{\phi}^{2}\right) u
$$




$$
=-\frac{2 M}{r^{2}} \partial_{t} u-\frac{2(r-M)}{r^{2}} \partial_{r} u-2 \cot \theta \partial_{\theta} u
$$

Although this wave equation is non-singular and can be reduced to symmetric hyperbolic form in the region $R_{1} \leq r \leq R_{2}$, its principle part, given by the left hand side of (1.3), has two notable features which complicate a numerical treatment:

- The appearance of the mixed $\partial_{t} \partial_{r}$ derivative. This is an effect of the Eddington-Finkelstein coordinates in which the $(r, \theta, \phi)=$ const are moving relative to the to the time slicing. In the standard ADM [5] formalism, this gauge effect is described as a non-vanishing shift, described by the shift vector $\beta^{\alpha}=\left(0, \beta^{r}, 0,0\right)$ where

$$
\beta^{r}=\frac{1}{1+\frac{r}{2 M}}
$$

- The change in sign of the coefficient of $\partial_{r}^{2}$ in passing across the black hole. This change in sign does not affect the hyperbolicity of the wave equation but it creates a horizon at $r=2 M$ across which waves cannot pass to the exterior. For $r>2 M$, the $(r, \theta, \phi)=$ const-observers move on timelike worldlines; for $r=2 M$, these worldlines are lightlike; and for $r<2 M$, they are spacelike. Thus, inside the horizon the $\partial_{t}$ direction becomes spacelike, i.e. an evolution based upon grid points with constant $r$ is superluminal inside the horizon.

These features are further complicated by the consideration of proper boundary conditions for a numerical treatment.

Here we study the numerical simulation of this problem by introducing a model 1-dimensional system which retains all the geometric features of the full problem. In the domain $0 \leq x \leq 1$, we consider the quasi-linear wave equation

$$
\begin{aligned}
\partial_{\alpha}\left(\sqrt{-g} g^{\alpha \beta} \frac{1}{\Phi} \partial_{\beta} \Phi\right) & =\partial_{t}\left(\frac{\sqrt{-g}}{\Phi} g^{t \beta} \partial_{\beta} \Phi\right)+\partial_{x}\left(\frac{\sqrt{-g}}{\Phi} g^{x \beta} \partial_{\beta} \Phi\right) \\
& =-\partial_{t}\left(\frac{1}{\Phi} \partial_{t} \Phi\right)+\partial_{t}\left(\frac{a}{\Phi} \partial_{x} \Phi\right)+\partial_{x}\left(\frac{a}{\Phi} \partial_{t} \Phi\right)+\partial_{x}\left(\frac{b-a^{2}}{\Phi} \partial_{x} \Phi\right) \\
& =0
\end{aligned}
$$

where the coefficients are smooth and satisfy $b>0$, and $a^{2}>b$ for $x=0$ and $a^{2}<b$ for $x=1$. These conditions ensure that the $t$-foliation is spacelike, that the metric determinant satisfies the Lorentzian requirement $g<0$, that the outer boundary $x=1$ is timelike and that the inner boundary $x=0$ is spacelike. The domain contains an event horizon at $a^{2}=b$ through which waves cannot cross in the outer direction. The shift vector has component $\beta^{x}=a$. For initial data satisfying $\Phi>0$, the Cauchy problem for this equation is well-posed.

The non-linearity is introduced in (1.6) in order to model another computational difficulty related to the full Einstein equations. When harmonic coordinates are introduced to fix the gauge freedom, the Einstein equations reduce to quasi-linear equations for the components of the metric whose principle part is identical to (1.5). In these harmonic coordinates, there exists pure gauge solutions (flat metrics)

$$
d s^{2}=\Phi\left(-d t^{2}+d x^{2}\right)+d y^{2}+d z^{2},
$$

where

$$
\Phi=1+f(t-x)
$$

which describing traveling waves with profile $f>-1$. The simulation of such gauge waves is complicated by the existence of exponentially growing gauge waves

$$
d s_{\lambda}^{2}=\Phi_{\lambda}\left(-d t^{2}+d x^{2}\right)+d y^{2}+d z^{2}
$$

where

$$
\Phi_{\lambda}=e^{\lambda t}(1+f(t-x))
$$

(for arbitrary $\lambda$ ) which can approximate the same Cauchy data. These exponentially growing solutions to the analytic problem can trigger a numerical instability, which cannot be damped by dissipative techniques. The simulation of (1.7) is one of the standardized tests proposed in the "Apples with Apples" project [6, 7], whose purpose is to compare different numerical treatments of the Einstein equations. A successful simulation of (1.7) requires controlling the excitation of (1.9) either by boundary conditions or by a conservation law on the finite difference system. The LSU 
group [8] has obtained excellent long term accuracy for the gauge wave test (with periodic boundaries) with a code that preserves a semi-discrete energy norm at the linearized level by means of the summation by parts method [9, 10, 11].

The quasi-linear wave equation (1.6) underlies this gauge wave problem 12]. Solutions of (1.6) can be used to construct gauge waves. For a background Minkowski metric, a non-singular solution of (1.6) is the traveling wave (1.8). The accurate long term simulation of this solution is complicated by the existence of the neighboring solutions $\Phi_{\lambda}$, given by (1.10), which grow exponentially in time, in precise analogy with the gauge wave problem.

We analyze the quasi-linear excision problem in terms of its elementary pieces. A successful algorithm for any initialboundary value problem must also be valid for the corresponding linearized problem with constant coefficients. This problem is considered in Sec. [II] We consider three separate regions: (i) behavior of the interior evolution, (ii) behavior at a time-like boundary and (iii) behavior at a spacelike boundary. Treatment of a time-like boundary in general relativity introduces yet another computational difficulty related to the constraints. In the harmonic formulation of the initial-boundary value problem, each component of the metric satisfies a quasi-linear linear wave equation whose principle part is identical to (1.2). In order for the solution to satisfy the harmonic constraints, it is necessary to apply a Dirichlet boundary condition to some metric components and a Neumann boundary condition to others [2]. For the purpose of future application of our results to harmonic evolution in numerical relativity, we concentrate here on Dirichlet and Neumann boundary conditions, although the treatment extends to the general maximally dissipative boundary condition.

Our spatial discretization of the wave equation is carried out in second differential order which has many advantages over first order formulations, as discussed in 13, 14]. These advantages have been recognized in recent second order harmonic approaches to numerical relativity [1, 2, 3]. For the case of zero shift, a comprehensive treatment of the wave equation in second order form has been given for both Dirichlet [17] and Neumann boundary conditions [18]. A major challenge of the excision problem is not only dealing with a shift but with a shift that is superluminal. This introduces complications even in the absence of boundaries. Standard implicit [15] and explicit [16] evolution algorithms for the second order wave equation with constant coefficients are unstable in the superluminal case. We discuss this in Sec. ПA where we give a finite difference algorithm for the subluminal case which is stable for both Dirichlet and Neumann boundary conditions. We give a detailed analysis, since there is little treatment of the second order wave equation with shift in the literature. Whereas treatment of the Dirichlet condition carries over unchanged, a non-zero shift forces a revision in the treatment of the Neumann condition. This algorithm for the subluminal case (the outer algorithm) is well suited for treating the outer boundary of the excision problem but becomes unstable inside the horizon, where the shift becomes superluminal. We present an alternative finite difference algorithm (the horizon algorithm) which is stable in this region and admits a stable spacelike excision boundary.

We establish stability by using the method of lines to reduce the wave equation to ordinary differential equations in time on a spatial grid and then applying two standard techniques: mode analysis and semi-discrete energy estimates supplied by the summation by parts technique [19]. The energy method is important because it can be extended to the wave equation with non-constant coefficients in a region with inner and outer boundaries. We use the energy associated with the $t$-direction along which the spatial grid propagates. However, inside the horizon where the $t$-direction becomes spacelike, this energy is effectively a component of momentum and does not provide a positivedefinite norm. Although it possible to introduce a positive-definite energy norm associated with the time-like normal to the Cauchy slicing [16], we do not pursue that direction here. Instead, we use mode analysis to establish the stability of the horizon algorithm and the excision algorithm at a spacelike boundary.

In Sec.III we treat the linearized excision problem. This involves blending together the outer and horizon algorithms obtained in Sec. III The additional ingredient is the necessity of non-constant coefficients in order to model a domain with spacelike inner boundary and timelike outer boundary. The homogeneous Dirichlet and Neumann boundary conditions are constructed so that the energy of the semi-discrete system is conserved when the coefficients of the wave operator are independent of time. In addition, the algorithm is constructed so that it conserves a monopole moment associated with the scalar field in the case of time-dependent coefficients.

The algorithm is implemented as a numerical code using a fourth order Runge-Kutta time integration. Two code tests of the linear excision problem are presented in Sec. [IIC] one where the data describes a pulse completely inside the horizon; the other where the data consists of an ingoing wave entering the outer boundary. The tests confirm the accuracy and stability predicted by the analysis of the finite difference algorithm.

In Sec. IV] we treat the excision problem for the quasi-linear wave equation. Although, semi-discrete energy conservation is not considered in the nonlinear case, we construct the algorithm so that the scalar monopole moment remains conserved. Studies of the gauge wave problem [12] show that either energy conservation or monopole conservation are sufficient to suppress the exponential mode (1.10). This is confirmed in the numerical tests of the quasi-linear excision problem presented in Sec. IVB

In Sec. $\mathbb{\nabla}$ we discuss the application to the harmonic Einstein equations of the algorithms developed here for the model excision problem. 


\section{ALGORITHMS FOR THE LINEAR WAVE EQUATION WITH CONSTANT COEFFICIENTS}

We consider the wave equation

$$
u_{t t}-2 a u_{x t}-\left(b-a^{2}\right) u_{x x}=0
$$

obtained from (1.6) by freezing the coefficients and making the linear approximation $\Phi \approx 1+u$. (Where no confusion arises, we use the abbreviated notation $\partial_{t} u=u_{t}$, etc.) We treat three separate problems

1. The interior Cauchy problem with both signs of $\left(b-a^{2}\right)$.

2. The initial-boundary value problem in the domain $0 \leq x \leq 1$ with $\left(b-a^{2}\right)>0$, and timelike boundaries at $x=0$ and $x=1$.

3. The initial-boundary value problem in the domain $0 \leq x<\infty$ with $\left(b-a^{2}\right)<0$, and a spacelike boundary at $x=0$.

Successful treatment of these three problems is necessary for the treatment of the full linear excision problem and guarantees that the rate of growth in the semi-discrete problem is bounded independent of the step size.

\section{A. The Cauchy problem}

The Cauchy problem for (2.1) is well-posed for every fixed value of $a$ and $b>0$. The Fourier mode $u=\hat{u} e^{i \omega x}$ satisfies

$$
\hat{u}_{t t}-2 i a \omega \hat{u}_{t}-\left(b-a^{2}\right) \omega^{2}=0
$$

with solution $\hat{u}=e^{\lambda t}$ where

$$
\lambda=i \omega(a \pm \sqrt{b})
$$

Thus $\Re(\lambda)=0$ and there are no exponentially growing modes

On a uniform spatial grid $x_{\nu}=\nu h$, consider the simplest second-order accurate finite difference approximation to (2.1),

$$
u_{t t}-2 a D_{0} u_{t}-\left(b-a^{2}\right) D_{+} D_{-} u=0
$$

where $D_{0} u_{\nu}=\left(u_{\nu+1}-u_{\nu-1}\right) /(2 h), D_{+} u_{\nu}=\left(u_{\nu+1}-u_{\nu}\right) / h$ and $D_{-} u_{\nu}=\left(u_{\nu}-u_{\nu-1}\right) / h$. The discrete Fourier mode $u_{\nu}=\hat{u} e^{i \omega \nu h}$ satisfies

$$
\hat{u}_{t t}-\frac{2 i a \sin (\omega h)}{h} \hat{u}_{t}+\frac{4\left(b-a^{2}\right) \sin ^{2}(\omega h / 2)}{h^{2}} \hat{u}=0
$$

with solution $\hat{u}=e^{\lambda t}$ where

$$
\lambda=\frac{i a \sin (\omega h)}{h} \pm \frac{i}{h} \sqrt{a^{2} \sin ^{2}(\omega h)+4\left(b-a^{2}\right) \sin ^{2}(\omega h / 2)} .
$$

For $b \geq a^{2}$ it follows that $\Re(\lambda)=0$ but for $b<a^{2}$ there are exponentially growing modes, e.g.

$$
\lambda= \pm \frac{2}{h} \sqrt{a^{2}-b}
$$

for the shortest wavelength mode $\omega h=\pi$.

In order to remedy this problem, we consider the alternative second order accurate finite difference approximation

$$
u_{t t}-2 a D_{0} u_{t}-\left(b D_{+} D_{-}-a^{2} D_{0}^{2}\right) u=0
$$

for which the discrete Fourier mode satisfies

$$
\hat{u}_{t t}-\frac{2 i a \sin (\omega h)}{h} \hat{u}_{t}+\left(\frac{4 b \sin ^{2}(\omega h / 2)-a^{2} \sin ^{2}(\omega h)}{h^{2}}\right) \hat{u}=0,
$$


with solution $\hat{u}=e^{\lambda t}$ where

$$
\lambda=\frac{i}{h}(a \sin (\omega h) \pm 2 \sqrt{b} \sin (\omega h / 2)) .
$$

Now $\Re(\lambda)=0$ for all values of the coefficients, subject to $b>0$.

Unfortunately, because the evolution algorithm (2.8) involves a wider stencil than (2.4) it is difficult to analyze its behavior and design a clean algorithm near a timelike boundary. Accordingly, we will use the outer algorithm (2.4) near a timelike boundary and the horizon algorithm (2.8) in a region extending from the excision boundary to the exterior of the horizon.

\section{B. Timelike boundary problem}

We treat the initial-boundary value problem for (2.1) in the domain $0 \leq x \leq 1$. For purposes of discussion, it suffices to concentrate on the boundary condition at $x=0$ for the choice $b-a^{2}=1$. Thus we consider the system

$$
u_{t t}-2 a u_{t x}-u_{x x}=0 .
$$

We can reduce this to the first order symmetric hyperbolic system

$$
\mathbf{u}_{t}:=\left(\begin{array}{l}
u \\
v
\end{array}\right)_{t}=\left(\begin{array}{cc}
2 a & 1 \\
1 & 0
\end{array}\right)\left(\begin{array}{l}
u \\
v
\end{array}\right)_{x}:=\mathbf{A} \mathbf{u}_{x}
$$

The eigenvalues of $\mathbf{A}$ are

$$
\kappa_{ \pm}=a \pm \sqrt{a^{2}+1}
$$

with eigenvectors

$$
\mathbf{e}_{ \pm}=\left(\begin{array}{c}
a \pm \sqrt{a^{2}+1} \\
1
\end{array}\right)
$$

Since $\kappa_{+}>0$ and $\kappa_{-}<0$, only one boundary condition is allowed at $x=0$.

The boundary conditions of interest for (2.11) give rise to an energy estimate via the conservation law

$$
\partial_{t} E=\left.F\right|_{x=1}-\left.F\right|_{x=0}
$$

where

$$
E=\frac{1}{2} \int_{0}^{1}\left(u_{t}^{2}+u_{x}^{2}\right) d x
$$

and

$$
F=u_{t}\left(u_{x}+a u_{t}\right)
$$

Because of the constraint problem in the harmonic initial-boundary value problem for Einstein's equation [2], we concentrate here on the the homogeneous Dirichlet boundary condition

$$
u_{t}=0
$$

and the homogeneous Neumann boundary condition

$$
u_{x}+a u_{t}=0,
$$

which satisfy $F=0$. These boundary conditions satisfy the dissipative condition $F \leq 0$ which guarantees a wellposed initial-boundary value problem. Note that the Neumann condition (2.19) involves the normal derivative $g^{x \alpha} \partial_{\alpha}=$ $\partial_{x}+a \partial_{t}$ in the rest frame intrinsic to the boundary.

One can also prove well-posedness by mode analysis. The half-space problem in the domain $0 \leq x<\infty$ is well posed if (2.11) has no solution

$$
u=e^{s t} \hat{u}(x),\|\hat{u}\|<\infty
$$


with $\Re(s)>0$, where $\hat{u}$ satisfies one of the boundary conditions (2.18) or (2.19). Introducing (2.20) into (2.11) gives

$$
s^{2}-2 a s \hat{u}_{x}-\hat{u}_{x x}=0 .
$$

Thus $\hat{u}=e^{\kappa x}$, where $\kappa$ solves the characteristic equation

$$
\kappa^{2}+2 a s \kappa-s^{2}=0
$$

with solutions

$$
\kappa_{ \pm}=s\left(-a \pm \sqrt{a^{2}+1}\right) .
$$

For $\Re(s)>0$, it follows that $\Re\left(\kappa_{+}\right)>0$ so that the general solution satisfying the norm requirement in (2.20) is

$$
\hat{u}(x)=\sigma e^{\kappa_{-} x}, \quad \Re\left(\kappa_{-}\right)<0 .
$$

Clearly $\sigma=0$ for the Dirichlet condition (2.18). Introducing (2.20) into the Neumann condition (2.19) gives

$$
\sigma\left(\kappa_{-}+a s\right)=-\sigma s \sqrt{a^{2}+1}=0 .
$$

Again $\sigma=0$ and there are no solutions with $\Re(s)>0$.

We consider now the difference approximation to 2.11),

$$
W_{\nu}:=u_{\nu t t}-2 a D_{0} u_{\nu t}-D_{+} D_{-} u_{\nu}=0,
$$

where $0 \leq \nu h \leq N h=1$, with one of the boundary conditions (2.18) or (2.19). For stability questions we can assume that the boundary condition is homogeneous.

We proceed in the same way as in the analytic case. Again we focus on the boundary condition at $x=0$ and consider the half-space problem $0 \leq \nu<\infty$. This problem is stable if [2.26) has no solution of type

$$
u_{\nu}=e^{s t} \hat{u}_{\nu}(s), \quad\|\hat{u}\|_{h}<\infty, \Re(s)>0 .
$$

Substitution of (2.27) into (2.26) gives

$$
s^{2} \hat{u}_{\nu}-2 a s D_{0} \hat{u}_{\nu}-D_{+} D_{-} \hat{u}_{\nu}=0 .
$$

Thus, $\hat{u}_{\nu}=\kappa^{\nu}$ where $\kappa$ solves the characteristic equation

$$
s^{2} h^{2}=\operatorname{ash}\left(\kappa-\frac{1}{\kappa}\right)+\frac{(\kappa-1)^{2}}{\kappa},
$$

i.e.

$$
(\kappa-1)^{2}+\left(\kappa^{2}-1\right) a s h-s^{2} h^{2} \kappa=0 .
$$

This equation for $\kappa$ has the two solutions

$$
\kappa_{ \pm}=\frac{s^{2} h^{2}+2 \pm s h \sqrt{s^{2} h^{2}+4\left(1+a^{2}\right)}}{2(1+a s h)} .
$$

The stability condition (2.27) requires that there are no modes with $\Re(s)>0$ and $|\kappa|<1$. We know from the stability of the Cauchy problem that there are no modes with $\Re(s)>0$ and $|\kappa|=1$. The limiting behavior as $s \rightarrow \infty$ is

$$
\kappa_{+} \sim \frac{s h}{a}
$$

and

$$
\kappa_{-} \sim-\frac{a}{s h}
$$

The limiting behavior as $s \rightarrow 0$ is

$$
\kappa_{ \pm} \rightarrow 1
$$


By continuity, the only possibility is $\left|\kappa_{-}\right|<1$ for $\Re(s)>0$.

Therefore the general solution of (2.27) is

$$
u_{\nu}=\sigma e^{s t} \kappa_{-}^{\nu} .
$$

Introduction of (2.35) into the Dirichlet condition (2.18), expressed on the grid as

$$
u_{0 t}=0
$$

gives $\sigma=0$. Thus the Dirichlet condition gives rise to a stable finite difference evolution.

The finite difference implementation of the Neumann condition is more complicated. We do not want to solve the half-space problem but the the strip problem $0 \leq x \leq 1, t \geq 0$, where we also need boundary conditions at $x=1$. It is true that if the corresponding left and right halfplane problems are stable, then the strip problem is also stable. However, slow growth in the error can be excited by multiple reflections between the boundaries. For the purpose of controlling this growth, in the interval $0 \leq \nu h \leq N h=1$, we introduce the semi-discrete energy norm

$$
E=h \sum_{1}^{N-1} \mathcal{E}+\frac{h}{4}\left(\left(u_{t N}\right)^{2}+\left(D_{-} u_{N}\right)^{2}+\left(u_{t 0}\right)^{2}+\left(D_{+} u_{0}\right)^{2}\right)
$$

where

$$
\mathcal{E}=\frac{1}{2} u_{t}^{2}+\frac{1}{4}\left(D_{+} u\right)^{2}+\frac{1}{4}\left(D_{-} u\right)^{2} .
$$

Note that this semi-discrete energy is constructed using one-sided differences. A semi-discrete energy could also be defined using central differences, which would be conserved if the evolution algorithm (2.26) were also replaced with central differences [12]. However, such a centered second order algorithm requires artificial dissipation to suppress the odd-even modes and the wide stencil complicates the formulation of boundary conditions. See [16] for further discussion.

A straightforward calculation gives

$$
\begin{aligned}
\mathcal{E}_{t}-u_{t} W & =a D_{0}\left(u_{t}^{2}\right)-\frac{a h}{2}\left(\left(D_{+} u_{t}\right)^{2}-\left(D_{-} u_{t}\right)^{2}\right) \\
& +\frac{1}{2}\left(u_{t} D_{-} D_{+} u+\left(D_{+} u_{t}\right) D_{+} u+u_{t} D_{+} D_{-} u+\left(D_{-} u_{t}\right) D_{-} u\right) .
\end{aligned}
$$

The summation of this expression can be reduced to boundary terms by means of the identities

$$
\begin{gathered}
h \sum_{1}^{N-1} D_{0} f=\frac{1}{2}\left(f_{N}+f_{N-1}\right)-\frac{1}{2}\left(f_{0}+f_{1}\right) \\
\sum_{1}^{N-1}\left(\left(D_{+} f\right) D_{+} g-\left(D_{-} f\right) D_{-} g\right)=\left(D_{-} f_{N}\right) D_{-} g_{N}-\left(D_{+} f_{0}\right) D_{+} g_{0}
\end{gathered}
$$

and

$$
h \sum_{1}^{N-1}\left(f D_{-} g+\left(D_{+} f\right) g\right)=f_{N} g_{N-1}-f_{1} g_{0} .
$$

After setting $W_{\nu}=0$ for $1 \leq \nu \leq N-1$, we obtain the semi-discrete version of energy conservation

$$
\begin{aligned}
\partial_{t} E & =\frac{1}{2} u_{t N}\left(h W_{N}+2 D_{0} u_{N}+a\left(u_{t(N+1)}+u_{t(N-1)}\right)\right) \\
& -\frac{1}{2} u_{t 0}\left(-h W_{0}+2 D_{0} u_{0}+a\left(u_{t 1}+u_{t(-1)}\right)\right) .
\end{aligned}
$$


Thus the Dirichlet conditions $u_{t N}=u_{t 0}=0$ are dissipative. We express the Neumann condition (2.19) in the second order accurate form

$$
\begin{aligned}
\mathcal{N}_{N}:=D_{0} u_{N}+\frac{a}{2}\left(u_{t(N+1)}+u_{t(N-1)}\right) & =q_{N} \\
\mathcal{N}_{0}:=D_{0} u_{0}+\frac{a}{2}\left(u_{t 1}+u_{t(-1)}\right) & =q_{0},
\end{aligned}
$$

where $q_{N}=q_{0}=0$ for homogeneous boundary data. These conditions involve the ghost points $\nu=-1$ and $\nu=N+1$ which we eliminate via the relations

$$
\begin{aligned}
\frac{h}{2} W_{N}+\mathcal{N}_{N} & =\frac{h}{2} u_{t t N}+D_{-} u_{N}+a u_{t(N-1)} \\
-\frac{h}{2} W_{0}+\mathcal{N}_{0} & =-\frac{h}{2} u_{t t 0}+D_{+} u_{0}+a u_{t 1} .
\end{aligned}
$$

Then, assuming that $W=O(h)$ at the boundary points, 2.45) gives a dissipative, second order accurate approximation to the Neumann boundary condition (2.19). We implement this boundary condition (2.45) to update the boundary points via

$$
\begin{array}{r}
u_{t t N}+\frac{2}{h}\left(D_{-} u_{N}+a u_{t(N-1)}-q_{N}\right)=0 \\
u_{t t 0}-\frac{2}{h}\left(D_{+} u_{0}+a u_{t 1}-q_{0}\right)=0
\end{array}
$$

where $q_{N}=q_{0}=0$ in the homogeneous case. This gives a stable problem in the strip for which the semi-discrete energy satisfies $E_{t}=0$ in the homogeneous case and is otherwise dictated by the boundary data.

Mode analysis of the halfplane problem confirms the stability of this Neumann condition. Introduction of (2.35) into (2.26) and (2.47) gives

$$
\begin{array}{r}
s^{2} h^{2} \kappa_{-}-a \operatorname{sh}\left(\kappa_{-}^{2}-1\right)-\left(\kappa_{-}-1\right)^{2}=0 \\
s^{2} h^{2}-2 a s h \kappa_{-}-2\left(\kappa_{-}-1\right)=0
\end{array}
$$

whose only solutions are

$$
\begin{aligned}
\tilde{\kappa}_{0} & =1 \\
\tilde{\kappa}_{ \pm} & =\frac{-1 \pm 2 i a \sqrt{1+a^{2}}}{1+2 a^{2}}
\end{aligned}
$$

which all satisfy $|\tilde{\kappa}|=1$. The corresponding frequencies are $s_{0}=0$ and

$$
s_{ \pm}= \pm \frac{2 i \sqrt{1+a^{2}}}{h} .
$$

In the continuum problem, a homogeneous Neumann boundary condition also leads to the conservation law of the monopole quantity

$$
Q=\int_{0}^{1}\left(u_{t}-a u_{x}\right) d x
$$

This carries over to the conservation of the semi-discrete monopole quantity

$$
\mathcal{Q}=h \sum_{\nu=1}^{N-1}\left(u_{t \nu}-a D_{0} u_{\nu}\right)+\frac{h}{2}\left(u_{t N}-a D_{-} u_{N}+u_{t 0}-a D_{+} u_{0}\right)
$$

i.e. $\mathcal{Q}_{t}=0$ when the homogeneous versions of (2.46) and (2.47) are satisfied. This semi-discrete monopole conservation can be extended to the non-linear problem, where it has proved effective at suppressing the long wavelength slowly growing mode (1.10). 


\section{Spacelike boundary problem $0 \leq x<\infty$.}

We now treat the initial-boundary value problem for (2.1) in the halfspace $0 \leq x<\infty$ for the case $a^{2}>b$. Since the boundary $x=0$ is spacelike, the characteristics leave the region and no boundary conditions are necessary in the continuum problem.

We use mode analysis with frozen coefficients to formulate a stable discretization near the boundary. Consider first the continuum system. Introduction of solutions of the form $u=e^{s t} \cdot \hat{u}(x)$ into (2.1) gives

$$
s^{2} \hat{u}-2 a s \hat{u}_{x}-\left(b-a^{2}\right) \hat{u}_{x x}=0 .
$$

This is an equation with constant coefficients and its solutions are of the form $\hat{u}=e^{\mu x}$ where $\mu$ are the solutions of the characteristic equation

$$
-s^{2}+2 a s \mu+\left(b-a^{2}\right) \mu^{2}=0
$$

i.e.

$$
\mu_{1,2}=-\frac{a s}{b-a^{2}} \pm \sqrt{\frac{a^{2} s^{2}}{\left(b-a^{2}\right)^{2}}+\frac{s^{2}\left(b-a^{2}\right)}{\left(b-a^{2}\right)^{2}}}=\frac{a s}{a^{2}-b} \pm \frac{s \sqrt{b}}{a^{2}-b} .
$$

Since $a>\sqrt{b}$, all roots satisfy $\Re\left(\mu_{1,2}\right)>0$ for $\Re(s)>0$. Thus the problem is well posed.

As difference approximation for the halfplane problem we use the horizon evolution algorithm (2.8), i.e.

$$
u_{\nu t t}-2 a \frac{\left(u_{\nu+1}-u_{\nu-1}\right)_{t}}{2 h}-b \frac{\left(u_{\nu+1}-2 u_{\nu}+u_{\nu-1}\right)}{h^{2}}+a^{2} \frac{\left(u_{\nu+2}-2 u_{\nu}+u_{\nu-2}\right)}{4 h^{2}}=0
$$

for $\nu=2,3, \ldots$. We need two extra boundary conditions to determine $u_{0}$ and $u_{1}$. We use the extrapolation conditions

$$
h^{3} D_{+}^{3} u_{0}=0, \quad h^{4} D_{+}^{4} u_{0}=0 .
$$

The algorithm is stable if there are no solutions of the type

$$
u_{\nu}=e^{s t} \kappa^{\nu},|\kappa|<1, \Re(s)>0 .
$$

Introduction of this into the evolution algorithm (2.8) gives the characteristic equation

$$
\tilde{s}^{2}-a \tilde{s}\left(\kappa-\frac{1}{\kappa}\right)-b\left(\kappa-2+\frac{1}{\kappa}\right)+\frac{a^{2}}{4}\left(\kappa-\frac{1}{\kappa}\right)^{2}=0
$$

for $\tilde{s}=h s$.

\section{Lemma}

(i) 2.61) has no solutions with

$$
|\kappa|=1 \quad \text { for } \Re(\tilde{s})>0 .
$$

(ii) There are exactly two solutions $\kappa_{1}, \kappa_{2}$ with

$$
\left|\kappa_{j}\right|<1, j=1,2 \quad \text { for } \Re(\tilde{s})>0 .
$$

(iii) For $\tilde{s} \rightarrow 0$,

$$
\lim _{\tilde{s} \rightarrow 0} \kappa_{1}=-\left(1-\frac{2 b}{a^{2}}\right)+\sqrt{\left(1-\frac{2 b}{a^{2}}\right)^{2}-1}, \quad \lim _{\tilde{s} \rightarrow 0} \kappa_{2}=-\left(1-\frac{2 b}{a^{2}}\right)-\sqrt{\left(1-\frac{2 b}{a^{2}}\right)^{2}-1} .
$$

Proof. Part (i) follows from the stability of the Cauchy problem, established in Sec. ЩA For $\tilde{s}$ real, as $\tilde{s} \rightarrow \infty$ the solutions of (2.61) with $|\kappa|<1$ converge to the solutions of

$$
\tilde{s}^{2}+\frac{a^{2}}{4} \frac{1}{\kappa^{2}}=0, \quad \text { i.e. } \quad \kappa_{1,2}= \pm \frac{i a}{2} \frac{1}{\tilde{s}} .
$$


Correspondingly, the solutions of (2.61) with $|\kappa|>1$ converge to the solutions of

$$
\tilde{s}^{2}+\frac{a^{2}}{4} \kappa^{2}=0, \quad \text { i.e. } \quad \kappa_{3,4}= \pm \frac{2 \tilde{s}}{i a} .
$$

Since the $4 \operatorname{roots} \kappa(\tilde{s})$ of (2.61) are continuous functions of $\tilde{s}$ and $|\kappa| \neq 1$ for $\Re(\tilde{s})>0$, there are exactly two roots $\kappa_{1,2}$ with $\left|\kappa_{1,2}\right|<1$ for all $\tilde{s}$ with $\Re(\tilde{s})>0$, thus establishing (ii). For $\tilde{s}=0$, we have

$$
-b \frac{(\kappa-1)^{2}}{\kappa}+\frac{a^{2}}{4} \frac{\left(\kappa^{2}-1\right)^{2}}{\kappa^{2}}=\frac{(\kappa-1)^{2}}{\kappa}\left(-b+\frac{a^{2}}{4} \frac{(\kappa+1)^{2}}{\kappa}\right)=0
$$

i.e.

$$
(\kappa-1)^{2}=0 \quad \text { or } \quad(\kappa+1)^{2}-\frac{4 b}{a^{2}} \kappa=\kappa^{2}+2\left(1-\frac{2 b}{a^{2}}\right) \kappa+1=0
$$

Therefore,

$$
\kappa_{1,2}=-\left(1-\frac{2 b}{a^{2}}\right) \pm \sqrt{\left(1-\frac{2 b}{a^{2}}\right)^{2}-1}, \quad \kappa_{3,4}=1 .
$$

A simple perturbation analysis shows that $\left|\kappa_{3,4}\right| \sim\left|e^{\mu_{1,2} h}\right|>1$ for $|\tilde{s}|<<1, \Re(\tilde{s})>0$, i.e. $\kappa_{3,4}$ correspond to the solution of (2.56). Since $a^{2}>b$, we have $\left|\kappa_{1,2}\right|=1$ for $\tilde{s} \rightarrow 0$ and (2.64) follows. This proves the lemma.

We want to show that the halfplane problem (2.58), 2.59) has no solutions

$$
u_{\nu}=e^{s t}\left(\sigma_{1} \kappa_{1}^{\nu}+\sigma_{2} \kappa_{2}^{\nu}\right) \quad \text { for } \quad \Re(s) \geq 0
$$

with $\left|\kappa_{1}\right|<1$ and $\left|\kappa_{2}\right|<1$. Introduction of (2.70) into the boundary conditions (2.59) gives

$$
\sigma_{1}\left(\kappa_{1}-1\right)^{3}+\sigma_{2}\left(\kappa_{2}-1\right)^{3}=0, \quad \sigma_{1}\left(\kappa_{1}-1\right)^{4}+\sigma_{2}\left(\kappa_{2}-1\right)^{4}=0 .
$$

Thus there are nontrivial solutions if and only if

$$
\left(\kappa_{1}-1\right)^{3}\left(\kappa_{2}-1\right)^{4}-\left(\kappa_{2}-1\right)^{3}\left(\kappa_{1}-1\right)^{4}=\left(\kappa_{1}-1\right)^{3}\left(\kappa_{2}-1\right)^{2}\left(\kappa_{2}-\kappa_{1}\right)=0 .
$$

By the lemma, $\kappa_{1} \neq 1, \kappa_{2} \neq 1$ for $\Re(s) \geq 0$. If $\kappa_{1}=\kappa_{2}$, then the general solution (2.70) is of the form

$$
u_{\nu}=e^{s t}\left(\sigma_{1} \kappa_{1}^{\nu}+\sigma_{2} \nu \kappa_{1}^{\nu}\right) \text {. }
$$

In this case (2.71) becomes

$$
\sigma_{1}\left(\kappa_{1}-1\right)^{3}+3 \sigma_{2} \kappa_{1}\left(\kappa_{1}-1\right)^{2}=0, \quad \sigma_{1}\left(\kappa_{1}-1\right)^{4}+4 \sigma_{2} \kappa_{1}\left(\kappa_{1}-1\right)^{3}=0,
$$

and there is a nontrivial solution if and only if

$$
\left(\kappa_{1}-1\right)^{6} \kappa_{1}=0
$$

Thus there are no such solutions.

\section{Time discretization}

The results in Sec's (IIB) and (IIC) prove that the outer algorithm gives a stable difference approximation for the halfplane problem with timelike boundary and that the horizon algorithm gives a stable difference approximation for the halfplane problem with a spacelike boundary. In Sec. (III), these results will be combined to give a stable difference approximation for the strip problem with spacelike inner boundary and timelike outer boundary.

For the time discretization, we use the method of lines. The spatial discretization reduces the problem to a large system of stable ODE's

$$
\mathbf{u}_{t t}=\frac{1}{h} \mathbf{A} \mathbf{u}_{t}+\frac{1}{h^{2}} \mathbf{B u}
$$


Introducing

$$
\mathbf{u}_{t}=\frac{1}{h} \mathbf{v}
$$

we obtain the first order system

$$
\left(\begin{array}{l}
\mathbf{v} \\
\mathbf{u}
\end{array}\right)_{t}=\frac{1}{h}\left(\begin{array}{cc}
\mathbf{B} & \mathbf{A} \\
\mathbf{I} & \mathbf{0}
\end{array}\right)\left(\begin{array}{l}
\mathbf{v} \\
\mathbf{u}
\end{array}\right) .
$$

We solve the system numerically using Runge-Kutta, 4th order. The time step is limited by the CFL condition determined from the Cauchy problem.

\section{THE LINEAR EXCISION PROBLEM}

We model a pulse of energy propagating into a horizon using the wave equation

$$
\left.-\partial_{t}^{2} u+\partial_{t}\left(a \partial_{x} u\right)+\partial_{x}\left(a \partial_{t} u\right)+\partial_{x}\left(\left(1-a^{2}\right) \partial_{x} u\right)\right)=0
$$

on the interval $0 \leq x \leq 1$, where we set

$$
\begin{aligned}
& a=\frac{5}{4}-x, \quad 0 \leq x \leq \frac{1}{2} \\
& a=\alpha(x), \quad \frac{1}{2} \leq x \leq \frac{3}{4} \\
& a=\frac{1}{2}, \quad \frac{3}{4} \leq x \leq 1,
\end{aligned}
$$

with $\alpha$ providing a smooth monotonic transition between the inner and outer regions. The underlying metric is Lorentzian and (3.1) is hyperbolic. The inner boundary is spacelike, the outer boundary is timelike and there is an event horizon at $x=1 / 4$.

The solution to (3.1) in the region $3 / 4 \leq x \leq 1$ has the exact form

$$
u=f\left(t+\frac{2}{3} x\right)+g(t-2 x)
$$

consisting of an ingoing wave $f$ and an outgoing wave $g$. Near the inner boundary all waves are ingoing, as can be see by freezing the coefficient $a$ to obtain the solution

$$
u=f\left(t+\frac{4}{9} x\right)+g(t+4 x)
$$

which is valid in the short wavelength limit.

Our goal is to simulate a wave $f$ incident on the boundary at $x=1$ which propagates across the horizon, while being partially backscattered. We also check that a pulse of compact support inside the horizon does not propagate into the exterior region.

\section{A. Linear algorithm with non-constant coefficients}

First we must modify the evolution-boundary algorithm to account for non-constant coefficients, as required for the excision problem. We consider the equation

$$
\left.-\partial_{t}^{2} u+\partial_{t}\left(a \partial_{x} u\right)+\partial_{x}\left(a \partial_{t} u\right)+\partial_{x}\left(c \partial_{x} u\right)\right)=0,
$$

where $a=a(t, x)$ and $c=c(t, x)$. For the case $a_{t}=c_{t}=0$, energy conservation (2.15) remains valid with the modifications

$$
E=\frac{1}{2} \int_{0}^{1}\left(u_{t}^{2}+c u_{x}^{2}\right) d x
$$


and

$$
F=u_{t}\left(c u_{x}+a u_{t}\right)
$$

With this generalization, the homogeneous Dirichlet boundary condition remains $u_{t}=0$ but the homogeneous Neumann condition (2.19) must be modified to the dissipative form

$$
c u_{x}+a u_{t}=0 .
$$

Note that for $c<0, E$ is not necessarily positive.

We modify the outer algorithm to

$$
W:=u_{t t}-\partial_{t}\left(a D_{0} u\right)-D_{0}\left(a u_{t}\right)-\frac{1}{2} D_{-}\left(\left(A_{+} c\right) D_{+} u\right)-\frac{1}{2} D_{+}\left(\left(A_{-} c\right) D_{-} u\right)=0,
$$

where

$$
A_{ \pm} f_{\nu}=\frac{f_{\nu \pm 1}+f_{\nu}}{2}
$$

The last two terms in (3.9) are equal because of the identity

$$
D_{-}\left(\left(A_{+} f\right) D_{+} g\right)=D_{+}\left(\left(A_{-} f\right) D_{-} g\right),
$$

but it is advantageous to express $W$ in a form which is manifestly reflection invariant.

Consider now semi-discrete energy conservation for the case $a_{t}=c_{t}=0$. We modify the semi-discrete energy (2.37) to

$$
E=h \sum_{1}^{N-1} \mathcal{E}+\frac{h}{4}\left(\left(u_{t N}\right)^{2}+\left(A_{-} c_{N}\right)\left(D_{-} u_{N}\right)^{2}+\left(u_{t 0}\right)^{2}+\left(A_{+} c_{0}\right)\left(D_{+} u_{0}\right)^{2}\right)
$$

where

$$
\mathcal{E}=\frac{1}{2} u_{t}^{2}+\frac{1}{4}\left(A_{+} c\right)\left(D_{+} u\right)^{2}+\frac{1}{4}\left(A_{-} c\right)\left(D_{-} u\right)^{2} .
$$

It follows that

$$
\begin{aligned}
\mathcal{E}_{t}-u_{t} W & =D_{0}\left(a u_{t}^{2}\right)-\frac{h}{2}\left(\left(D_{+} u_{t}\right) D_{+}\left(a u_{t}\right)-\left(D_{-} u_{t}\right) D_{-}\left(a u_{t}\right)\right) \\
& +\frac{1}{2} u_{t} D_{-}\left(\left(A_{+} c\right) D_{+} u\right)+\frac{1}{2}\left(D_{+} u_{t}\right)\left(A_{+} c\right) D_{+} u \\
& +\frac{1}{2} u_{t} D_{+}\left(\left(A_{-} c\right) D_{-} u\right)+\frac{1}{2}\left(D_{-} u_{t}\right)\left(A_{-} c\right) D_{-} u
\end{aligned}
$$

Following the procedure leading to (2.43), the summation of this expression gives the semi-discrete flux conservation law

$$
\begin{aligned}
\partial_{t} E & =\frac{1}{2} u_{t N}\left(h W_{N}+\left(A_{+} c_{N}\right) D_{+} u_{N}+\left(A_{-} c_{N}\right) D_{-} u_{N}+\left(A_{+} a_{N}\right) u_{t(N+1)}+\left(A_{-} a_{N}\right) u_{t(N-1)}\right) \\
& -\frac{1}{2} u_{t 0}\left(-h W_{0}+\left(A_{+} c_{0}\right) D_{+} u_{0}+\left(A_{-} c_{0}\right) D_{-} u_{0}+\left(A_{+} a_{0}\right) u_{t 1}+\left(A_{-} a_{0}\right) u_{t(-1)}\right) .
\end{aligned}
$$

We express the Neumann condition (3.8) in the second order accurate form

$$
\mathcal{N}^{\prime}:=\frac{1}{2}\left(\left(A_{+} c\right) D_{+} u+\left(A_{-} c\right) D_{-} u+\left(A_{+} a\right) T_{+} u_{t}+\left(A_{-} a\right) T_{-} u_{t}\right)=0,
$$

where $T_{ \pm} f_{\nu}=f_{\nu \pm 1}$. At the boundaries, $\mathcal{N}_{N}^{\prime}$ and $\mathcal{N}_{0}^{\prime}$ contain ghost points outside the computational domain, which we eliminate via the relations

$$
\begin{aligned}
\frac{h}{2} W_{N}+\mathcal{N}_{N}^{\prime} & =\frac{h}{2} u_{t t N}+\left(A_{-} c_{N}\right) D_{-} u_{N}+\left(A_{-} a_{N}\right) u_{t(N-1)} \\
-\frac{h}{2} W_{0}+\mathcal{N}_{0}^{\prime} & =-\frac{h}{2} u_{t t 0}+\left(A_{+} c_{0}\right) D_{+} u_{0}+\left(A_{+} a_{0}\right) u_{t 1} .
\end{aligned}
$$


Then, requiring that the wave equation be satisfied at the boundary points, i.e. that $W_{N}=W_{0}=0$, the conservation law (3.15) implies that the Neumann boundary conditions

$$
\begin{array}{r}
\mathcal{N}_{N}^{\prime}:=\frac{h}{2} u_{t t N}+\left(A_{-} c_{N}\right) D_{-} u_{N}+\left(A_{-} a_{N}\right) u_{t(N-1)}=0 \\
\mathcal{N}_{0}^{\prime}:=-\frac{h}{2} u_{t t 0}+\left(A_{+} c_{0}\right) D_{+} u_{0}+\left(A_{+} a_{0}\right) u_{t 1}=0
\end{array}
$$

are dissipative.

Energy conservation holds in the continuum theory only when the coefficients of the wave operator are time independent. However, in the continuum problem, conservation of the monopole quantity (2.53) holds for general time-dependent coefficients in the case of homogeneous Neumann boundary conditions. This carries over to the conservation of the semi-discrete quantity

$$
\mathcal{Q}=h \sum_{\nu=1}^{N-1}\left(u_{t \nu}-a D_{0} u_{\nu}\right)+\frac{h}{2}\left(u_{t N}-a D_{-} u_{N}+u_{t 0}-a D_{+} u_{0}\right)
$$

when the Neumann condition (3.16) has the correction

$$
\mathcal{N}:=\mathcal{N}^{\prime}+\frac{h^{2}}{4} a_{t} D_{+} D_{-} u=0
$$

which preserves second order accuracy. As a result of this correction, (3.19) and (3.20) are modified to

$$
\begin{aligned}
& \frac{h}{2} W_{N}+\mathcal{N}_{N}:=\frac{h}{2}\left(u_{t t N}-a_{t N} D_{-} u_{N}\right)+\left(A_{-} c_{N}\right) D_{-} u_{N}+\left(A_{-} a_{N}\right) u_{t(N-1)}=0 \\
& -\frac{h}{2} W_{0}+\mathcal{N}_{0}:=-\frac{h}{2}\left(u_{t t 0}-a_{t 0} D_{+} u_{0}\right)+\left(A_{+} c_{0}\right) D_{+} u_{0}+\left(A_{+} a_{0}\right) u_{t 1}=0 .
\end{aligned}
$$

Setting $W_{\nu}=0$ for $1 \leq \nu \leq N-1$, a straightforward calculation then gives

$$
\mathcal{Q}_{t}=\frac{h}{2} W_{N}+\mathcal{N}_{N}+\frac{h}{2} W_{0}-\mathcal{N}_{0}
$$

Thus, again requiring that $W_{N}=W_{0}=0$, the boundary conditions

$$
\begin{array}{r}
\mathcal{N}_{N}=\frac{h}{2}\left(u_{t t N}-a_{t N} D_{-} u_{N}\right)+\left(A_{-} c_{N}\right) D_{-} u_{N}+\left(A_{-} a_{N}\right) u_{t(N-1)}=0 \\
\mathcal{N}_{0}=-\frac{h}{2}\left(u_{t t 0}-a_{t 0} D_{+} u_{0}\right)+\left(A_{+} c_{0}\right) D_{+} u_{0}+\left(A_{+} a_{0}\right) u_{t 1}=0
\end{array}
$$

imply $\mathcal{Q}_{t}=0$. Thus we have established that $\mathcal{Q}_{t}=0$ in the general case and $E_{t}=0$ when the coefficients are frozen in time.

Finally, in the inner region, we modify the horizon algorithm (2.8) to the non-constant coefficient form

$$
V:=u_{t t}-\partial_{t}\left(a D_{0} u\right)-D_{0}\left(a u_{t}\right)-\frac{1}{2} D_{-}\left(\left(A_{+} b\right) D_{+} u\right)-\frac{1}{2} D_{+}\left(\left(A_{-} b\right) D_{-} u\right)+D_{0}\left(a^{2} D_{0} u\right)=0,
$$

where $c=b-a^{2}$.

\section{B. Blending the outer and horizon algorithms}

For the purpose of excision, we need a prescription for switching from the outer algorithm $W=0$ to the horizon algorithm $V=0$. We introduce a smooth, monotonic blending function $f(c)=f\left(b-a^{2}\right)$, satisfying $f=1$ for $c \leq 0$ and $f=0$ for $c \geq 1 / 2$. Referring to (3.9) and (3.28), we then use the blended evolution algorithm $B=0$, where

$$
B=W-D_{-}\left(\left(A_{+}\left(f a^{2}\right)\right) D_{+} u\right)+D_{0}\left(f a^{2} D_{0} u\right)=W+\frac{h^{2}}{4} D_{+} D_{-}\left(f a^{2} D_{+} D_{-} u\right),
$$

which reduces to $W$ near the outer boundary and to $V$ inside the horizon. 
When $\partial_{t} g^{\alpha \beta}=0$, the blended algorithm satisfies a semi-discrete energy conservation law. For periodic boundary conditions (identifying the points $\nu=0$ and $\nu=N$ ), this takes the form $\partial_{t} E_{B}=0$ where

$$
E_{B}=h \sum_{1}^{N} \mathcal{E}_{B}
$$

with

$$
\mathcal{E}_{B}=\mathcal{E}+\frac{f h^{2} a^{2}}{8}\left(D_{+} D_{-} u\right)^{2} .
$$

For the boundary conditions of the excision problem, the energy (3.12) is modified in the same way,

$$
E_{B}=E+h \sum_{1}^{N-1} \frac{f h^{2} a^{2}}{8}\left(D_{+} D_{-} u\right)^{2} .
$$

Since the support of the blending function $f$ is isolated from the outer timelike boundary, the semi-discrete energy flux through the outer boundary remains given by the first term in (3.15). The inner excision boundary is inside the horizon where, in the continuum theory, the energy density is not necessarily positive. As a result, the energy flux across the spacelike inner boundary can have either sign, in contrast to the situation that would hold for a spacelike boundary outside the horizon.

\section{Tests of the linear excision algorithm}

In order to validate the linear algorithm a set of test-runs was performed where the outer boundary condition at $x=1$ was either Dirichlet or Neumann, while at $x=0$ the extrapolation condition (4.4) was applied. The gridstep was chosen to be $h=1 /(\rho \cdot 2000), \rho=1,2,4$ with the time-step set to $\Delta t=h / 10$. The background coefficients were set according to (3.2) with $\alpha(x)=245 / 4-513 x+1728 x^{2}-2880 x^{3}+2368 x^{4}-768 x^{5}$ such that a $C^{2}$ smoothness results.

In the first set of runs the initial data at $t=0$ was a $C^{3}$ pulse of compact support inside the horizon with $u_{t}(0, x)=0$ and

$$
\begin{aligned}
& u(0, x)=0.5 \times[4 \xi(1-\xi)]^{4} \quad \text { for } \quad x_{0} \leq x \leq x_{0}+0.2 \\
& u(0, x)=0 \quad \text { elsewhere, }
\end{aligned}
$$

with $\xi(\lambda)=(\lambda-0.025) / 0.2$. Homogeneous Dirichlet data $u(t, 1)=0$ was given at the outer boundary. At the initial location of the pulse inside the horizon, both characteristics point to the left. The condition $u_{t}(0, x)=0$ implies that the initial pulse is the superposition of two pulses, whose amplitudes have opposite signs, which propagate along these two characteristics. This is clearly seen in Fig. 1 where at first the "faster" mode (with small negative amplitude) falls into the excised region, while the "slower" mode (with positive amplitude) propagates in the same direction. We also monitor the convergence factor for the error modes that have propagated outside the horizon, defined in terms of the $L_{2}$ norm over the interval $x_{H}=0.25 \leq x \leq 1$ as

$$
\mathcal{C}\left(x \geq x_{H}\right)=\log _{2}\left(\frac{\left\|u_{\rho=1}\right\|_{2}}{\left\|u_{\rho=2}\right\|_{2}}\right) .
$$

Second order accuracy would imply $\mathcal{C}\left(x \geq x_{H}\right) \geq 2$ since the analytic solution vanishes outside the horizon.

In this region outside the horizon, the simulation consists purely of short wavelength error. At first there is a high convergence rate but after about 6 crossing times the short wavelength error is not resolved on the grid. The addition of dissipation to the evolution scheme greatly extends the time for which convergence holds in the outer region, as shown in Fig. 2] Dissipation is added by modifying (2.78) according to

$$
\begin{array}{r}
v_{t} \rightarrow v_{t}-\epsilon_{v} D_{+} D_{-} \rho D_{+} D_{-} v \\
u_{t} \rightarrow u_{t}-\epsilon_{u} D_{+} D_{-} \rho D_{+} D_{-} u,
\end{array}
$$

where we set $\epsilon_{v}=\epsilon_{u}=0.1 \cdot h^{3}$ and $\rho$ is a smooth function with $\rho=b$ in the interior and $\rho=0$ near both boundaries. 

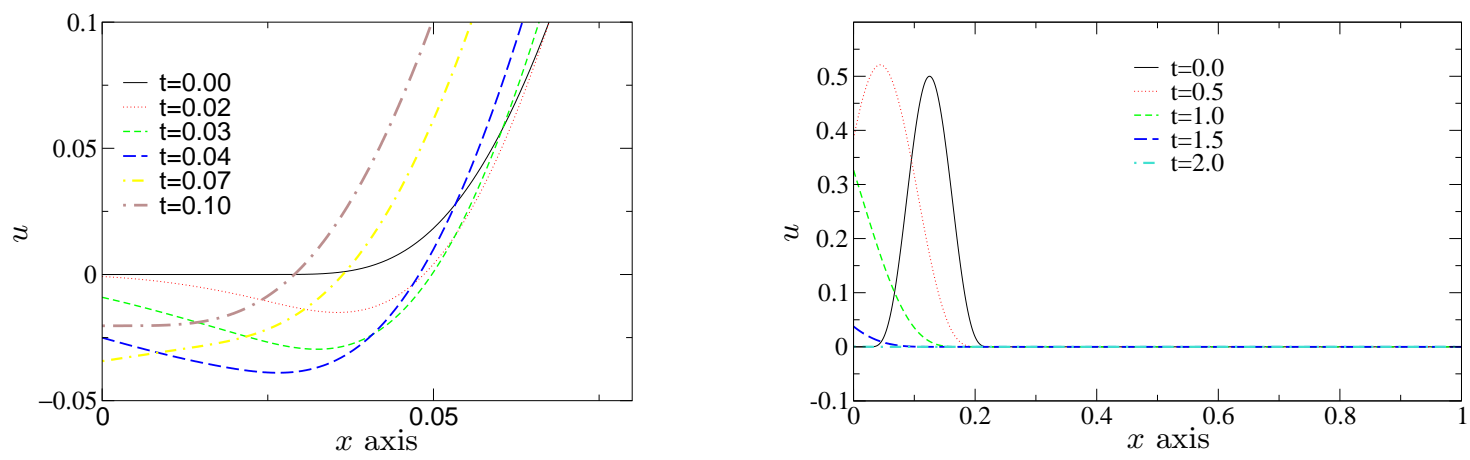

FIG. 1: Sequence of snapshots showing the initial pulse falling into the excised region. The left graph shows the early behavior as the faster mode (with negative amplitude) propagates through the excision boundary. The right graph shows the slower mode making its way through the excision boundary, with no observable trace remaining at $t=2.0$.

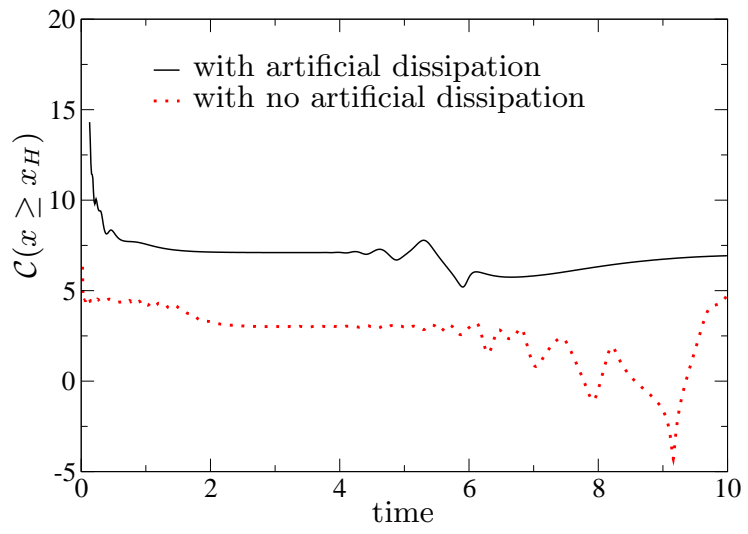

FIG. 2: Plot of the convergence factor $\mathcal{C}\left(x \geq x_{H}\right)$ as a function of time for the test run with an initial pulse inside the horizon. It is evident that the addition of dissipation is necessary for long term convergence.

In the second set of runs, the initial Cauchy data was set to zero, $u(0, x)=u_{t}(0, x)=0$, and a wave was introduced through the outer boundary $x=1$ by prescribing inhomogeneous Neumann data

$$
\left.g^{x \alpha} \partial_{\alpha} u\right|_{x=1}=\left.\left(\frac{3}{4} u_{x}+\frac{1}{4} u_{t}\right)\right|_{x=1}=q(t) .
$$

Figure 3 shows snapshots of the evolution for a wave generated by boundary data $q(t)$ consisting of a single pulse. The pulse enters the outer boundary in the incoming mode of (3.3) with characteristic velocity $-3 / 2$, propagates across the blending region and leaves the grid at the excision boundary. The figure also shows the remnant signal when no dissipation is added to the code. After the initial pulse has entered the outer boundary, the Neumann data is homogeneous and reflects any signal propagating to the right but signals propagating to the left can leave the system across the excision boundary. At $t=1$, short wavelength noise fills the region inside the horizon. By $t=5$, the short wavelength error has either propagated through the excision boundary or has been converted into longer wavelengths. At $t=10$, no visible signal remains. The short wavelength noise at $t=1$ can be effectively eliminated by a small amount of dissipation.

We checked long term global convergence by prescribing a periodic wave entering though the outer boundary with the inhomogeneous Neumann data

$$
q(t)=0, \quad \text { for } t<1 / 150
$$



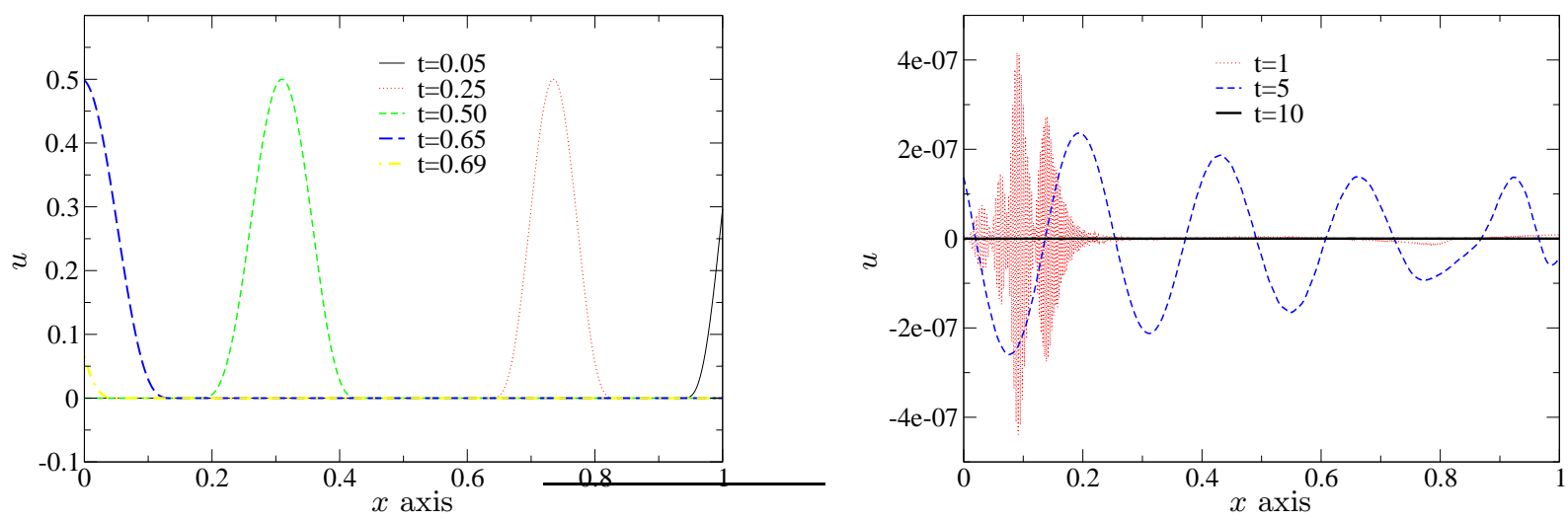

FIG. 3: Sequence of snapshots showing a pulse propagating across the grid. The left graph shows the propagation of the main signal, while the right graph illustrates the behavior of the residual modes. The short wavelength error can be eliminated by dissipation.
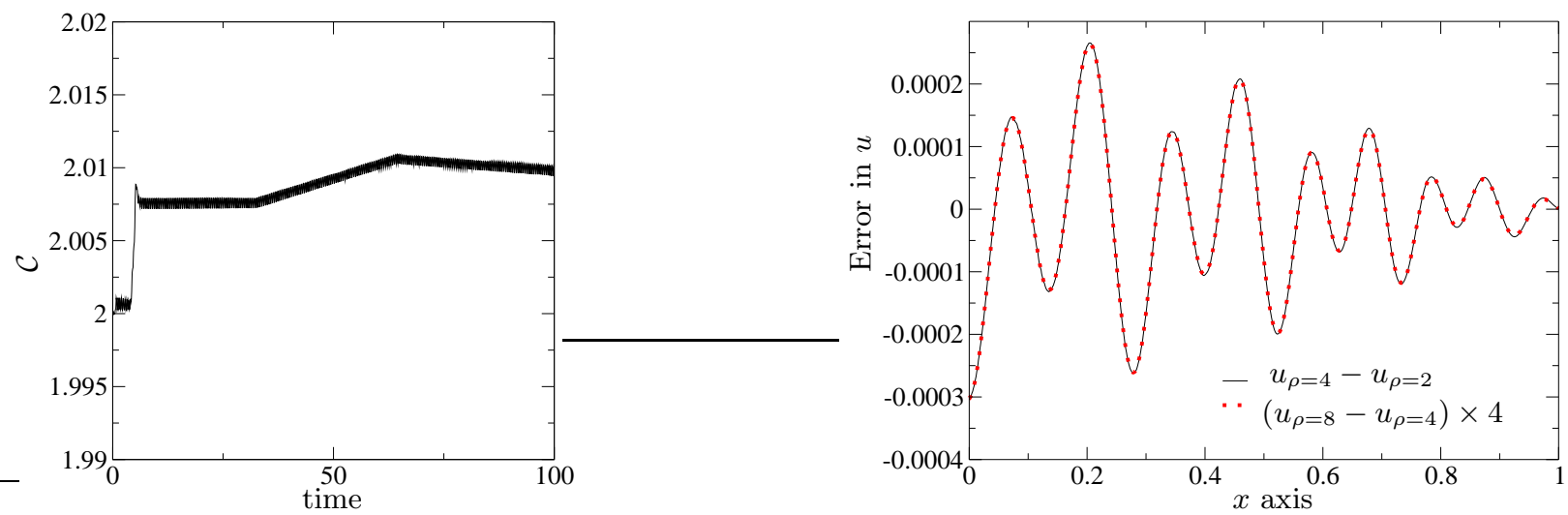

FIG. 4: Convergence plots for the test run with a periodic wave introduced through the outer (timelike) boundary. On the left, the plot of the convergence factor $\mathcal{C}$ vs. time indicates good second order convergence up to the end of the simulation at $t=100$. The quality of the long term performance is reinforced by the right graph which shows that, at the end of the simulation, the rescaled error profiles are in nearly perfect agreement. No artificial dissipation was introduced.

$$
q(t)=\left.\left(c \partial_{x}+a \partial_{t}\right)\left[A \sin ^{4}(\pi[x-1.01+1.5 t])\right]\right|_{x=1}, \text { for } \quad t \geq 1 / 150
$$

Again the initial Cauchy data was set to zero. The $C^{3}$ boundary data (3.38) is initially set to zero to provide $C^{\infty}$ consistency with the Cauchy data. No artificial dissipation was added in this test.

We measured Cauchy convergence of the numerical solution by monitoring the convergence factor

$$
\mathcal{C}=\log _{2}\left(\frac{\left\|u_{\rho=1}-u_{\rho=2}\right\|_{2}}{\left\|u_{\rho=2}-u_{\rho=4}\right\|_{2}}\right) .
$$

For the given data and grid sizes, the code displays second order accuracy to within less than one percent. Figure 4 plots the convergence factor as well as a snapshot of the residual finite difference error at the end of the simulation, at $t=100$. 


\section{THE QUASI-LINEAR EXCISION PROBLEM}

\section{A. Algorithms for the quasi-linear wave equation}

We extend our algorithms to the quasi-linear wave equation (1.6) in such a way that the outer algorithm continues to obey a semi-discrete monopole conservation law in the case of homogeneous Neumann boundary conditions. We again set $c=b-a^{2}$ and treat the general case of time-dependent, non-constant coefficients. We generalize the outer algorithm (3.9) to the finite-difference form

$$
W:=\partial_{t}\left(\frac{1}{\Phi} \Phi_{t}\right)-\partial_{t}\left(\frac{a}{\Phi} D_{0} \Phi\right)-D_{0}\left(\frac{a}{\Phi} \Phi_{t}\right)-\frac{1}{2} D_{-}\left(\left(A_{+} \frac{c}{\Phi}\right) D_{+} \Phi\right)-\frac{1}{2} D_{+}\left(\left(A_{-} \frac{c}{\Phi}\right) D_{-} \Phi\right)=0 .
$$

and generalize the horizon algorithm (3.28) to

$$
V:=\partial_{t}\left(\frac{1}{\Phi} \Phi_{t}\right)-\partial_{t}\left(\frac{a}{\Phi} D_{0} \Phi\right)-D_{0}\left(\frac{a}{\Phi} \Phi_{t}\right)-\frac{1}{2} D_{-}\left(\left(A_{+} \frac{b}{\Phi}\right) D_{+} \Phi\right)-\frac{1}{2} D_{+}\left(\left(A_{-} \frac{b}{\Phi}\right) D_{-} \Phi\right)+D_{0}\left(\frac{a^{2}}{\Phi} D_{0} \Phi\right)=0 .
$$

We blend these algorithms together using the quasi-linear version of (3.29), i.e $B=0$ with

$$
B=W-D_{-}\left(\left(A_{+} \frac{f a^{2}}{\Phi}\right) D_{+} \Phi\right)+D_{0}\left(\frac{f a^{2}}{\Phi} D_{0} \Phi\right)=W+\frac{h^{2}}{4} D_{+} D_{-}\left(\frac{f a^{2}}{\Phi} D_{+} D_{-} \Phi\right),
$$

As the boundary condition for the horizon algorithm at the spacelike excision boundary, we use the extrapolation conditions (4.4), now written as

$$
h^{3} D_{+}^{3} \Phi_{0}=0, \quad h^{4} D_{+}^{4} \Phi_{0}=0 .
$$

Similarly, as homogeneous Dirichlet condition for the outer algorithm at a timelike outer boundary we retain the linear form $\Phi_{t}=0$. We formulate the homogeneous Neumann condition for the outer algorithm to establish semi-discrete monopole conservation corresponding to the continuum conservation law

$$
\partial_{t} \int_{0}^{1}\left(\frac{\Phi_{t}}{\Phi}-\frac{a \Phi_{x}}{\Phi}\right) d x=0
$$

We proceed as follows.

For periodic boundary conditions, (4.1) implies the semi-discrete conservation law $\partial_{t} \mathcal{Q}=0$ where

$$
\mathcal{Q}=h \sum_{\nu}\left(\frac{\Phi_{t \nu}}{\Phi_{\nu}}-\frac{a_{\nu} D_{0} \Phi_{\nu}}{\Phi_{\nu}}\right)
$$

This conservation law can be extended to a homogeneous Neumann boundary condition by adding boundary terms to (4.6) in the form

$$
\begin{aligned}
\mathcal{Q} & =h \sum_{\nu=1}^{N-1}\left(\frac{\Phi_{t \nu}}{\Phi_{\nu}}-\frac{a_{\nu} D_{0} \Phi_{\nu}}{\Phi_{\nu}}\right) \\
& +\frac{h}{2}\left(\frac{\Phi_{t 0}}{\Phi_{0}}-\frac{a_{0} D_{+} \Phi_{0}}{\Phi_{0}}+\frac{\Phi_{t N}}{\Phi_{N}}-\frac{a_{N} D_{-} \Phi_{N}}{\Phi_{N}}\right) .
\end{aligned}
$$

We generalize the Neumann condition (3.16) to the non-linear form

$$
\mathcal{N}:=\frac{1}{2}\left(\left(A_{+} \frac{c}{\Phi}\right) D_{+} \Phi+\left(A_{-} \frac{c}{\Phi}\right) D_{-} \Phi+\left(A_{+} \frac{a}{\Phi}\right) T_{+} \Phi_{t}+\left(A_{-} \frac{a}{\Phi}\right) T_{-} \Phi_{t}\right)+\frac{h^{2}}{4} \partial_{t}\left(\frac{a}{\Phi}\right) D_{+} D_{-} \Phi=0 .
$$

As a result of the nonlinearities, (3.23) and (3.24) are modified to

$$
\begin{aligned}
\frac{h}{2} W_{N}+\mathcal{N}_{N}: & =\frac{h}{2}\left(\partial_{t}\left(\frac{\Phi_{t N}}{\Phi_{N}}\right)-\partial_{t}\left(\frac{a_{N}}{\Phi_{N}}\right) D_{-} \Phi_{N}\right)+\left(A_{-} \frac{c_{N}}{\Phi_{N}}\right) D_{-} \Phi_{N}+\left(A_{-} \frac{a_{N}}{\Phi_{N}}\right) \Phi_{t(N-1)} \\
- & \frac{h}{2} W_{0}+\mathcal{N}_{0}:=-\frac{h}{2}\left(\partial_{t}\left(\frac{\Phi_{t 0}}{\Phi_{0}}\right)-\partial_{t}\left(\frac{a_{0}}{\Phi_{0}}\right) D_{+} \Phi_{0}\right)+\left(A_{+} \frac{c_{0}}{\Phi_{0}}\right) D_{+} \Phi_{0}+\left(A_{+} \frac{a_{0}}{\Phi_{0}}\right) \Phi_{t 1}
\end{aligned}
$$



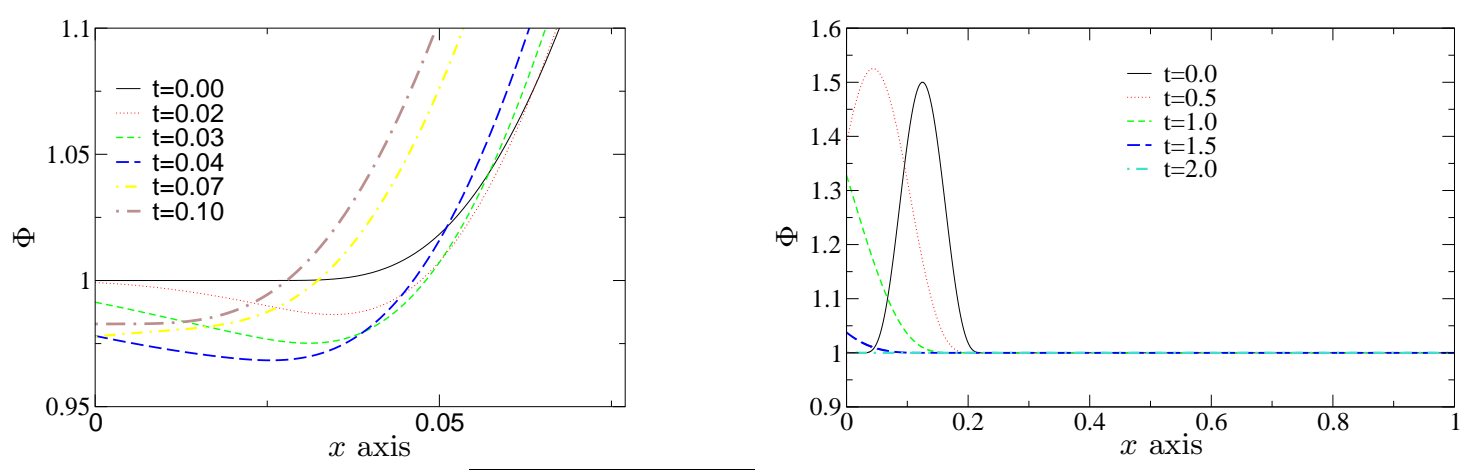

FIG. 5: Sequence of snapshots showing the initial pulse falling into the excised region, evolved with the quasi-linear system. The left graph shows the faster mode (with negative amplitude), while the right graph shows the slower mode making its way through the excision boundary. Grid-resolution for this simulation was $h=1 / 8000$.

Setting $W_{\nu}=0$ for $1 \leq \nu \leq N-1$, a straightforward calculation leads to the nonlinear version of [3.25]), i.e.

$$
\mathcal{Q}_{t}=\frac{h}{2} W_{N}+\mathcal{N}_{N}+\frac{h}{2} W_{0}-\mathcal{N}_{0}
$$

We implement the homogeneous Neumann condition in the form

$$
\begin{gathered}
\mathcal{N}_{N}=\frac{h}{2}\left(\partial_{t}\left(\frac{\Phi_{t N}}{\Phi_{N}}\right)-\partial_{t}\left(\frac{a_{N}}{\Phi_{N}}\right) D_{-} \Phi_{N}\right)+\left(A_{-} \frac{c_{N}}{\Phi_{N}}\right) D_{-} \Phi_{N}+\left(A_{-} \frac{a_{N}}{\Phi_{N}}\right) \Phi_{t(N-1)}=0 \\
\mathcal{N}_{0}=-\frac{h}{2}\left(\partial_{t}\left(\frac{\Phi_{t 0}}{\Phi_{0}}\right)-\partial_{t}\left(\frac{a_{0}}{\Phi_{0}}\right) D_{+} \Phi_{0}\right)+\left(A_{+} \frac{c_{0}}{\Phi_{0}}\right) D_{+} \Phi_{0}+\left(A_{+} \frac{a_{0}}{\Phi_{0}}\right) \Phi_{t 1}=0
\end{gathered}
$$

Then the requirement that $W_{N}=W_{0}=0$ leads to semi-discrete monopole conservation $\mathcal{Q}_{t}=0$.

\section{B. Tests of the quasi-linear excision algorithm}

The tests performed with the quasi-linear code were based upon the ones performed with the linear code, with the same Cauchy data or boundary data now prescribed for $(\Phi-1)$ as was prescribed for the linearized field $u$ in Sec. IIIC

The initial data for the first set of runs consisted of a pulse of compact support inside the horizon with homogeneous Dirichlet data at the outer boundary. The initial peak amplitude of the pulse (3.33) was $\Phi_{\max }=1.5$, putting it in the nonlinear regime. Since the characteristic speeds determined by the background metric are the same as in the linearized case, the snapshots of the quasi-linear evolution shown in Fig. [5 are qualitatively similar to the linearized ones in Fig. [1 There is no visible propagation of the signal into the region outside the horizon. The convergence factor $\mathcal{C}\left(x \geq x_{H}\right)$ for the error modes that have propagated outside the horizon is defined by replacing $u$ by $(\Phi-1)$ in (3.34). As in the linear case, the short wavelength error in the exterior region is unresolved on the grid. The plots in Fig. [6] show that dissipation must be added to the code to obtain long term convergence.

In the second set of non-linear runs, the initial Cauchy data was set to zero and a wave introduced through the outer boundary by prescribing inhomogeneous Neumann data. Figure 7 shows snapshots of the evolution for a wave consisting of a single pulse entering the outer boundary. No artificial dissipation was used. The main signal behaves qualitatively similar to the linearized case in Figure 3 but the error modes behave differently. At $t=1$ the short wavelength error again fills the region inside the horizon but now long wavelength error has also been excited which extends to the outer boundary. This corresponds to mixing in the exponential modes

$$
\Phi=e^{\lambda\left(t-\frac{2 x}{3}\right)},
$$

which are exact solutions (for any $\lambda$ ) in the region $x \geq 3 / 4$ to the quasi-linear wave equation (3.1). These modes are consistent with the homogeneous Neumann boundary condition (3.37) in effect after the pulse has entered the 


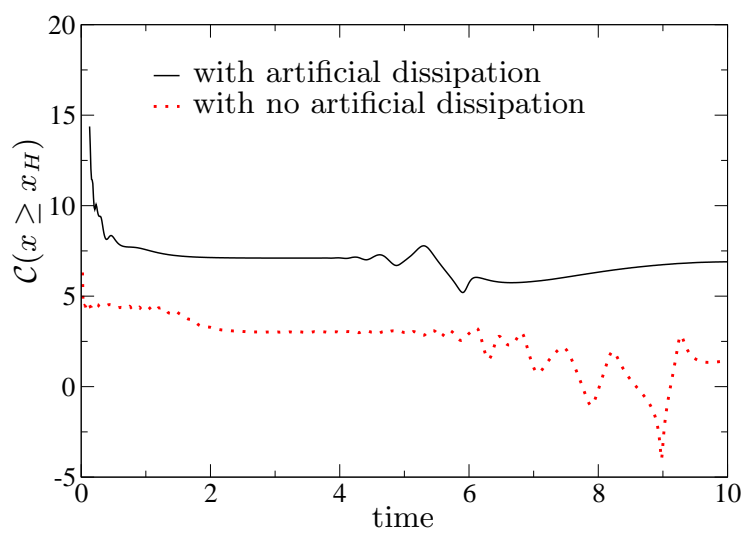

FIG. 6: Plot of the convergence factor $\mathcal{C}\left(x \geq x_{H}\right)$ vs. time. for the non-linear test run for an initial pulse inside the horizon. Addition of dissipation is necessary for long term convergence.
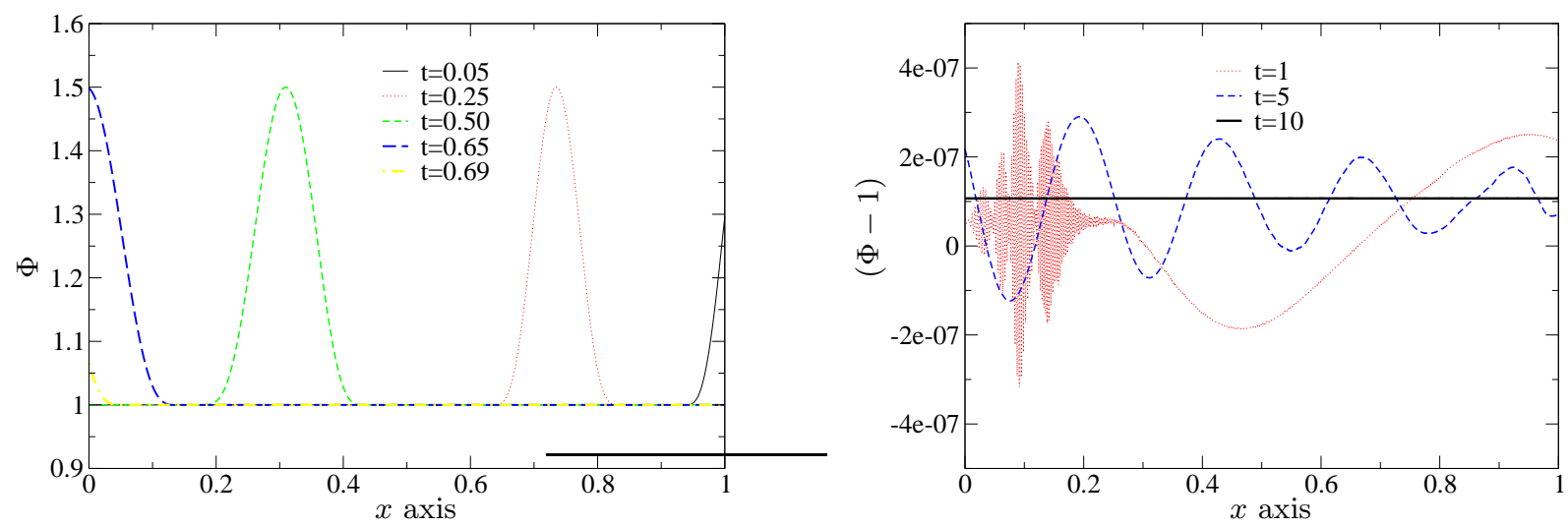

FIG. 7: Sequence of snapshots showing a pulse propagating across the grid with resolution $h=1 / 2000$. The left graph shows the propagation of the signal itself, while the right graph illustrates the behavior of the residual modes. The long wavelength mode, which is apparent at $t=1$, decays by $t=5$ and no visible signal remains at $t=10$.

system and $q(t$ vanishes. However, the mode (4.14) is not excited with positive $\lambda$ and decays by $t=5$. No visible signal remains at $t=10$. Thus the discrete monopole conservation built into the quasi-linear system is effective at suppressing exponentially growing long wavelength modes.

A further test of long term performance is the introduction of a periodic wave through the outer boundary by prescribing the inhomogeneous Neumann data (3.38). We measured Cauchy convergence by monitoring the convergence factor $\mathcal{C}$ (3.39) applied to the numerical solution for $\Phi$. Figure 8 plots $\mathcal{C}$ as well as a snapshot of the residual finite difference error at $t=100$, when the simulation was ended. The code displays second order accuracy to better than 1 percent. No artificial dissipation was used in the test.

\section{DISCUSSION}

We have based successful simulations of the model excision problem for a 1-dimensional quasi-linear wave equation on evolution and boundary algorithms which were proved to be stable in the linear case. The quasi-linear algorithm incorporates a conserved scalar monopole moment which suppresses the excitation of the long wavelength exponentially growing modes, such as (4.14), which are latent in the system when Neumann boundary conditions are used. The 

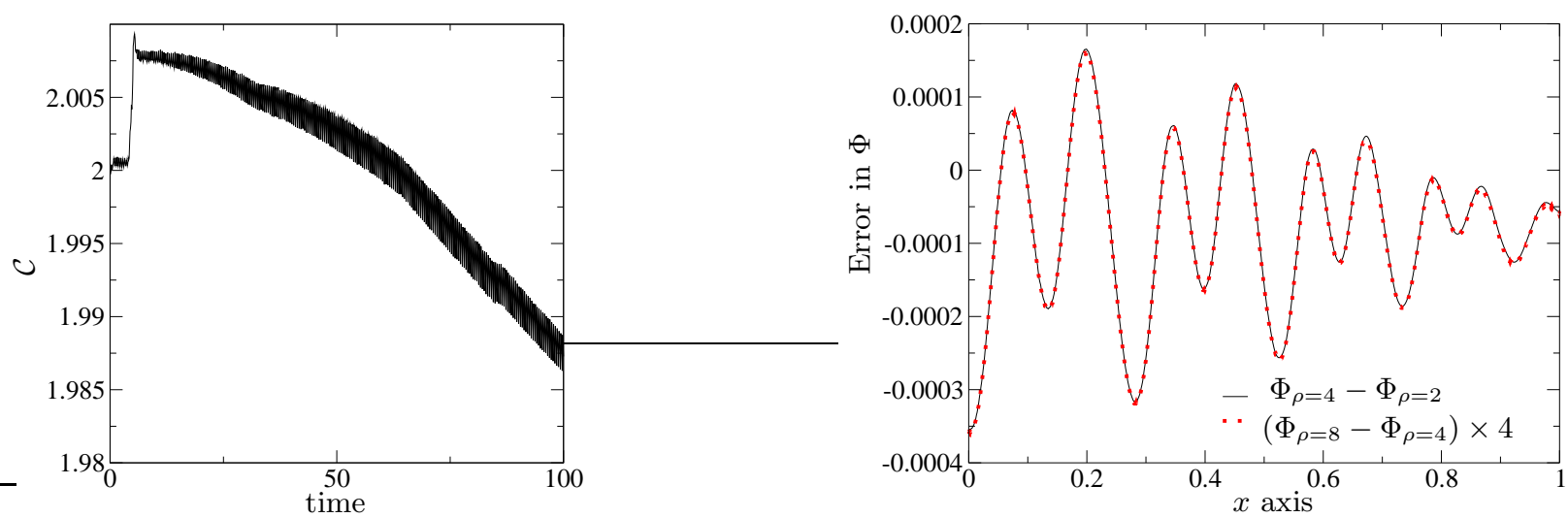

FIG. 8: Convergence plots for the non-linear test run with periodic pulse introduced through the outer (timelike) boundary. On the left, the time dependence of the convergence factor $\mathcal{C}$ indicates good second oder convergence. This result is reinforced by the right graph where, at the end of the simulation, at $t=100$, the rescaled error profiles show a nearly perfect agreement. No artificial dissipation was used in this test.

global excision strategy involves matching an exterior evolution algorithm to a horizon algorithm for evolution of the interior region. The exterior algorithm admits simple implementation of an outer boundary condition and it obeys a discrete version of flux-energy conservation which guarantees stability. Inside the horizon, this energy is not positive-definite and the exterior algorithm is unstable. The horizon algorithm is stable in the interior region and admits a stable extrapolation algorithm to update the spacelike excision boundary. The choice of horizon algorithm presented here is based upon centered differencing. Other choices are possible in the model 1-dimensional problem. In particular, a horizon algorithm based completely upon one-sided differencing is stable and requires no extrapolation at the inner boundary. This would be a very attractive feature if it could be extended to the higher dimensional problem.

Preliminary investigations indicate that the computational algorithms presented here can be taken over in a fairly straightforward manner to the harmonic formulation of the full 3-dimensional gravitational problem. In that case the Einstein equations reduce to to the quasi-linear form

$$
\frac{1}{\sqrt{-g}} \partial_{\alpha}\left(\sqrt{-g} g^{\alpha \beta} \partial_{\beta} g^{\mu \nu}\right)=S^{\mu \nu}
$$

where the $S^{\mu \nu}$ consist of lower differential order terms which do not contribute to the principle part. These equations have a well-posed initial-boundary value problem when the boundary data is explicitly prescribed [2].

The principle part of (5.1) can be treated in the same way as a quasi-linear wave equation (1.2) for each component of the metric. From the point of view of designing a code, the two main differences from our model excision problem are (i) the the 3-dimensional nature of the full problem and (ii) the constraints which enter into the boundary conditions.

The evolution algorithms readily extend to 3-dimensions. When $S^{\mu \nu}$ is neglected, the flux conservative form of (5.1) can be carried over to the 3 dimensional discretized system to obtain discrete analogues of monopole conservation for each metric component. Furthermore, when the metric coefficients in the wave operator are frozen, semi-discrete energy conservation results for each metric component in the case of periodic boundary conditions. With a cubic grid boundary, the results for the quasi-linear wave equation generalize to dissipative Dirichlet and Neumann boundary conditions for the metric components. The only essential complication is the application of Neumann conditions at the edges and corners of the cube. Here among possible approaches are the use of the summation by parts technique, as in [10], or the introduction of a smooth boundary which is treated by interpolation, as in [17, 18].

The constraints on the boundary data present another complication. The constraints on the system (5.1), which guarantee that the solutions satisfy Einstein's equations, are the harmonic coordinate conditions

$$
\partial_{\nu}\left(\sqrt{-g} g^{\mu \nu}\right)=\hat{H}^{\mu}
$$

where $\hat{H}^{\mu}$ are explicit harmonic driving functions. These constraints are satisfied by the solutions of (5.1) if a certain mixture of Dirichlet and Neumann boundary conditions are used for the metric components [2]. Then the constraints 
are satisfied when homogeneous boundary data are given and the initial-boundary problem for Einstein's equations is well-posed. In the case of inhomogeneous constraint-preserving boundary data it is not known whether the system remains well posed.

It should be emphasized that, in addition to the computational difficulty, there are analytic and geometric problems in treating black holes. There is the possibility of exponentially growing perturbations to the analytic problem in the inner region near the excision boundary. There is the nonlocal nature of the true event horizon. Although the spacelike apparent horizon can be traced out as the evolution proceeds, it is impossible to locate the null event horizon until the exterior evolution is complete. The matching of an inner horizon algorithm to an outer algorithm must be carried out across an artificial horizon which results from a given choice of shift, as in our model problem. In the black hole excision problem, such an artificial horizon could be defined in terms of the hypersurface across which the the evolution goes from superluminal to subluminal.

Pretorius [3] has recently obtained promising results using a second order harmonic code to simulate a black hole by means of excision. We are now in the process of applying the new techniques presented here to this problem.

\section{Acknowledgments}

We thank M. Babiuc, L. Lehner and M. Tiglio for their input. This work was supported by the National Science Foundation under grant PH-0244673 to the University of Pittsburgh.

[1] D. Garfinkle, Phys.Rev., D65, 044029 (2002).

[2] B. Szilágyi and J. Winicour, Phys. Rev., D68, 041501 (2003).

[3] F. Pretorius, "Numerical relativity using a generalized harmonic decomposition", gr-qc/0407110

[4] "Novel finite-differencing techniques for numerical relativity: application to black hole excision", G. Calabrese, L. Lehner, D. Neilsen, J. Pullin, O. Reula, O. Sarbach, and M. Tiglio, gr-qc/0302072

[5] R. Arnowitt, S. Deser and C. Misner, in Gravitation: An Introduction to Current Research, ed. L. Witten (New York, Wiley, 1962).

[6] www.appleswithapples.org

[7] M. Alcubierre, et al, Class. Quantum Grav., 21, 589 (2004).

[8] "3D simulations of Einstein's equations: symmetric hyperbolicity, live gages and dynamic control of the constraints", M. Tiglio, L. Lehner, D. Neilsen, gr-qc/0312001

[9] H.-O. Kreiss and G. Scherer, SIAM J. Numer. Anal. 29, 640 (1992).

[10] "Summation by parts and dissipation for domains with excised regions", G. Calabrese, L. Lehner, O. Reula, O. Sarbach, and M. Tiglio, gr-qc/0308007

[11] "The discrete energy method in numerical relativity: Towards long term stability", L. Lehner, D. Neilsen, O. Reula and M. Tiglio, gr-qc/0312001

[12] "Some mathematical problems in numerical relativity", M. Babiuc, B. Szilágyi and J. Winicour, gr-qc/0404092

[13] H.-O. Kreiss, N. A. Peterson and J. Yström, SIAM J. Numer. Anal. 40, 1940 (2002).

[14] H.-O. Kreiss and O. E. Ortiz, Lect. Notes Phys., 604, 359 (2002).

[15] M. Alcubierre and B. Schutz, J. Comput. Phys., 112, 44 (1994).

[16] "Finite differencing second order systems describing black hole spacetimes", G. Calabrese, gr-qc/0410062

[17] "A second order accurate embedded boundary method for the wave equation with Dirichlet data", H.-O. Kreiss and N. A. Peterson, preprint UCRL-JRNL-202686, Lawrence Livermore National Lab (2004).

[18] "Difference approximations of the Neumann problem for the second order wave equation", H.-O. Kreiss, N. A. Peterson and J. Yström, preprint UCRL-JC-153184, Lawrence Livermore National Lab (2003).

[19] B. Gustafsson, H.-O. Kreiss and J. Oliger, Time Dependent Problems and Difference Methods (Wiley, NY, 1995). 